\title{
Mesoscale Resolution Radar Data Assimilation Experiments with the Harmonie Model
}

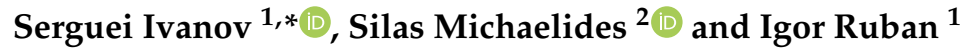 \\ Odessa State Environmental University (OSENU), 15, Lvovska Str., 65016 Odessa, Ukraine; iggru@i.ua \\ 2 The Cyprus Institute, 20, Konstantinou Kavafi Str., Aglantzia, 2121 Nicosia, Cyprus; s.michaelides@cyi.ac.cy \\ * Correspondence: svvivo@te.net.ua; Tel.: +38-050-631-3655
}

Received: 7 August 2018; Accepted: 7 September 2018; Published: 11 September 2018

\begin{abstract}
This study presents a pre-processing approach adopted for the radar reflectivity data assimilation and results of simulations with the Harmonie numerical weather prediction model. The proposed method creates a 3D regular grid in which a horizontal size of meshes coincides with the horizontal model resolution. This minimizes the representative error associated with the discrepancy between resolutions of informational sources. After such preprocessing, horizontal structure functions and their gradients for radar reflectivity maintain the sizes and shapes of precipitation patterns similar to those of the original data. The method shows an improvement of precipitation prediction within the radar location area in both the rain rates and spatial pattern presentation. It redistributes precipitable water with smoothed values over the common domain since the control runs show, among several sub-domains with increased and decreased values, correspondingly. It also reproduces the mesoscale belts and cell patterns of sizes from a few to ten kilometers in precipitation fields. With the assimilation of radar data, the model simulates larger water content in the middle troposphere within the layer from $1 \mathrm{~km}$ to $6 \mathrm{~km}$ with major variations at $2.5 \mathrm{~km}$ to $3 \mathrm{~km}$. It also reproduces the mesoscale belt and cell patterns of precipitation fields.
\end{abstract}

Keywords: harmonie model; radar data assimilation; pre-processing; mesoscale precipitation patterns

\section{Introduction}

Precipitation plays an important role in both the water cycle and energy balance of the atmosphere. However, due to the high spatial and temporal variability of precipitation in the mesoscale, obtaining accurate quantitative precipitation estimates is still a "first-line frontier" task. Moreover, the Global Precipitation Measurement (GPM) mission has shown notable differences in estimations of precipitation obtained from various platforms especially for low rain rates [1]. Current rain-gauge networks mainly suffer from sparse distribution and limited coverage [2]. Although satellite-based infrared and visible data have high spatial resolution, relations between the radiance from the cloud and precipitation are indirect and non-unique [3]. Additionally, the evaluations of satellite quantitative precipitation estimates have some limitations in terms of the deficiency in the observations, the evaluation methodology, the selection of time windows for evaluation, and the short periods for evaluation [4]. Passive microwave instruments provide acceptable estimates of precipitation. Nevertheless, they exhibit low spatial and temporal resolution [5]. The advantage of the ground-based radar network in Europe is the high spatiotemporal resolution. This is the reason to make a choice in favor of such data for predicting mesoscale precipitation patterns and associated atmospheric variables.

The Operational Program for the Exchange of Weather Radar Information (OPERA) was launched for improving the harmonization of radars and their measurements [6]. The program provides three composite products such as instantaneous surface rain rate, instantaneous maximum reflectivity, 
and one-hour rainfall accumulation. Gathered data includes three-dimensional volumes of reflectivity and radial wind. When assimilated in convection-permitting models, radar data could yield significant improvements in the representation of the spatial distribution of cloud and precipitation as well as rain rates [7-9]. This is achieved due to the fact that ground-based radars have a high spatial and temporal resolution and provide a three-dimensional volume of information. It is worth noting that radar data are heterogeneously distributed by providing abundant information near radar locations while such information becomes sparser with an increasing distance from the sensor. Further improvement of assimilation systems is seen in the optimization of pre-processing radar data in terms of homogenizing data coverage along with increasing confidence in them by averaging stochastic errors.

The objective of this study is to present a pre-processing approach adopted for the radar reflectivity data assimilation and the results of simulations with the Harmonie numerical weather prediction model. Through a case study, it will be shown that the method can improve the prediction of precipitation within the radar location area both in terms of the rain rates and spatial pattern presentation.

Even though radars measure the reflectivity and radial wind, this study focuses at the former parameter only. Quality control and other extra technical procedures are also omitted by assuming that they have been performed at preliminary steps during the initial processing of radar data. Further pre-processing is performed in different ways and may include various methods. Among these is the spatial screening of the raw data over some pixels in pursuit of both to reject abundant data and rid horizontal correlations in observation errors. The screening includes either filtering or smoothing procedures. The former is an easy way to routinely perform thinning of high-resolution radar data such as selection data of every predefined bin along a ray. Simple thinning of abundant radar observations throws a significant part of data out from the process, but it also affects the spectral distribution of sub-scales finer than a thinning parameter. An alternative to the thinning is the averaging of observations within a given box to create a new value located at an average position. This also allows us to average out random observation errors [10]. This method, which is called superobbing, is used for remote sensing observations such as atmospheric motion vectors and Doppler radar radial wind measurements [11]. With this approach, the observation (O) minus the background (B) differences (denoted as $\mathrm{O}-\mathrm{B}$ ) or innovations are averaged. For each observation, its model simulated analogue is computed and an O-B innovation is calculated. These innovations are then averaged and added to the model observation closest to the center of the superobbing cell to provide the super-observation. Optimization of the superobbing processing from dense raw data is the compromise between the above two factors including saving finer than average size scales and obtaining more stable estimates for the remaining scales by reducing stochastic errors [12]. Additionally, the problem of representativeness is necessary to be accounted for while comparing and assimilating data from sources with different spatial resolutions. Experience with assimilating high-resolution data shows that such data coverage may provide unsatisfactory results. In particular, tests with the Generalized Cross-Validation (GCV) method [13] using a simulated high-resolution data set at full resolution has led to a poorer analysis than a lower resolution data set that preceded it. The larger the nonconformity between the resolution of the observations network is, on the one hand, and that of the model, on the other hand, the larger the numerical impact on the result is or, equivalently, the larger the representativeness error is.

A modified method of pre-processing is presented in Section 2 in which the model and data used in this study are elaborated on. Results of simulations with the proposed pre-processing approach are presented in Section 3. Section 4 presents the discussion and concluding remarks.

\section{Materials and Methods}

\subsection{Pre-Processing of Radar Data}

In this study, the data assimilation system in the convection-permitting Harmonie model is further developed by involving radar reflectivity measurements. The focus is on optimizing the inner parameters of the pre-processing procedure rather than a data assimilation algorithm itself. 
In pursuing the compatibility between the model resolution and smoothed radar observation density, the "cube-smoothing" approach is proposed. It seems obvious to optimally feed the model with observations at the same resolution as the model grid. Similar approaches at the assimilating step are explored for simulated reflectivity in a 1D $+3 \mathrm{D}$ variational assimilation system by feeding vertical profiles of reflectivity on a regular grid [14], an Ensemble Kalman Filter Analysis (EnKF) [15], and a Hybrid EnKF-3Dvar algorithm [16]. The earlier method performs backward retrieving of radar reflectivity from atmospheric microphysics variables for further assimilating measurements. This approach is operationally used in the model and pursuits the aim to globally assimilate radar data by doing it with a coarser resolution than explored in the regional model. The two latter approaches were developed within the Advanced Regional Prediction System (ARPS) and verified with the use of an Observing System Simulation Experiment (OSSE). However, as previously mentioned [13], the involvement of simulated high-resolution data sets in assimilating can, under certain circumstances, lead to an improper analysis.

The proposed pre-processing method for smoothing radar reflectivity data imposes a minor additional computational cost, which can be afforded before the model is run and tuned for a specific domain. The method first creates a 3D regular grid in which a horizontal size of meshes coincides with a horizontal model resolution. This should minimize the error associated with the discrepancy between resolutions of informational sources [17] while the impact from filtering out sub-grid scales may occur. This is seen from comparing structure functions for original radar reflectivity data and those calculated after pre-processing (Figure 1). A Structure function as an alternative statistic to view variations in time series or fields is often used in meteorology $[18,19]$ and is denoted by the equation below.

$$
D_{A A}(L)=\frac{1}{N} \sum_{k=0}^{N-1}\left[A_{k}-A_{k+j}\right]^{2}
$$

where $L=r=j \Delta r$ represents the spatial separation, $r$, between the two measurements. The advantage of the structure function compared to other statistics is in its low dependence on variations between ensemble members as well as the possibility for obtaining stable estimates on the basis of a single limited length series containing systematic errors. For zero separation distance, the structure function is identical to zero and increases with the increasing separation until it reaches its maximum value, i.e., when it saturates. Such a distance implies lack of connectivity in a field and serves as a criterion for dominating scales in atmospheric processes. Regarding the slow changing structure functions for most atmospheric variables, the gradient of the function allows for the identification of a point on the curve, which corresponds to this criterion.

To estimate horizontal size and shape of precipitation patterns in the original data and after pre-processing with various smoothing values, the structure functions for radar reflectivity have been obtained. Only measurements from lower bins have been taken into account on the assumption that it provides horizontal scales in precipitation fields. Figure 1 shows that the saturation of the structure function starts at a few kilometer distance. Smoothing with $0.5 \mathrm{~km}$ yields very similar results both in the value and behaviors of the original data as the gradient. The latter allows us to clearly specify changes in the function behavior. In particular, saturation of the structure functions occurs at the shortest lag of about $1 \mathrm{~km}$, which confirms the dominant role of mesoscales. Smoothing with the 2.5 and $5 \mathrm{~km}$ shows weaker gradients at the smallest resolved scales but also background (secondary) phenomena appear at the scales of about 20 to $30 \mathrm{~km}$. This numerical (artificial) effect, which results from the inconsistency between filtering parameters and existent atmospheric patterns, is one of the reasons leading to a systematic phase error in the modeling of precipitation [20]. All structure functions completely saturate above the $60 \mathrm{~km}$ lag where the large-scale circulation dominates.

The drawback even for complete radar data sets is that they fill-in only a part of the physical or model spaces. For instance, the six Finish radars used in this study cover from $5 \%$ to about $20 \%$ of a model grid depending on the horizontal smoothing parameter and the vertical factor at the pre-processing step (Figure 2). 


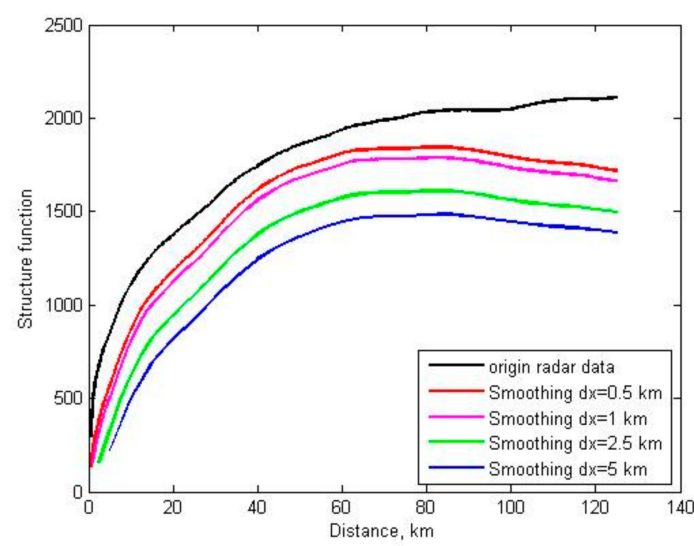

(a)

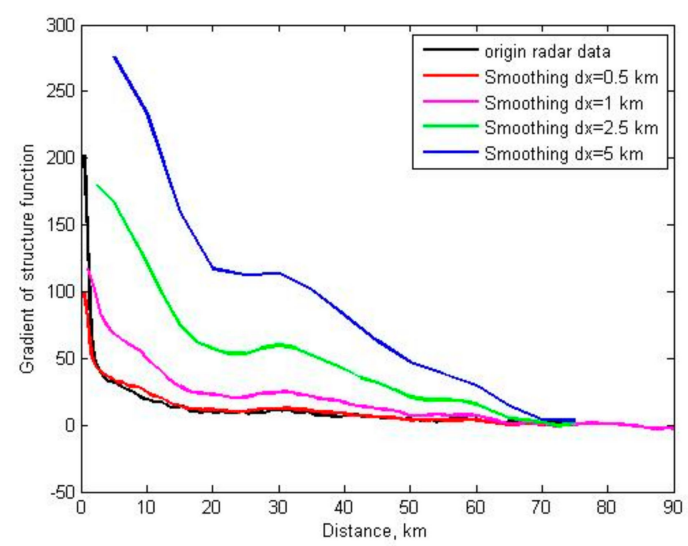

(b)

Figure 1. (a) Structure functions and (b) their gradients of radar reflectivity measurements for original data and after pre-processing with various smoothing parameters.

This is a considerable difference between the OSSE approach and the real data sets. A vertical size for meshes can be chosen on the basis of several criteria. Among the most appropriate ones are those that correspond to a list of model layers with varying steps along the vertical coordinate to homogeneous filling-in meshes by radar measurements and to a regular vertical step. Regarding the irregularly distributed Finnish radar network, the preliminary estimates of filling-in 3D-meshes have been obtained for the whole domain in one batch (Figure 3). The use of the vertical step in accordance to the Harmonie model level list leads to minor filling-in of lower layers, which significantly increases. Otherwise, the regular but rather large vertical step of $500 \mathrm{~m}$ yields an opposite distribution of the data. So far, the equal filling-in approach seems to ensure the homogeneous saturation of the model grid, which was selected for further pre-processing. At least three direct impacts from the pre-processing smoothing are clear. Over-smoothing with a parameter larger than the model's resolution leads to underestimating the total amount of water content and the small decreases but intensive cells of precipitation as well as the incorrectly presenting spatial patterns of rain water in the atmosphere. To this end, these factors affect the initial conditions and disrupt the subsequent evolution of precipitation patterns. Smoothing with $0.5 \mathrm{~km}$ yields reflectivity distributions similar to the original (Figure 2a).

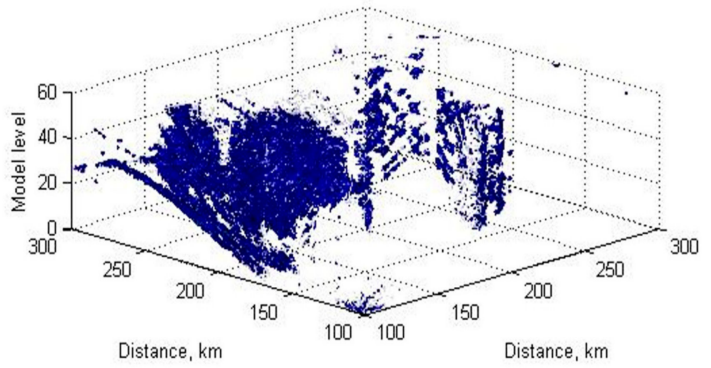

(a)

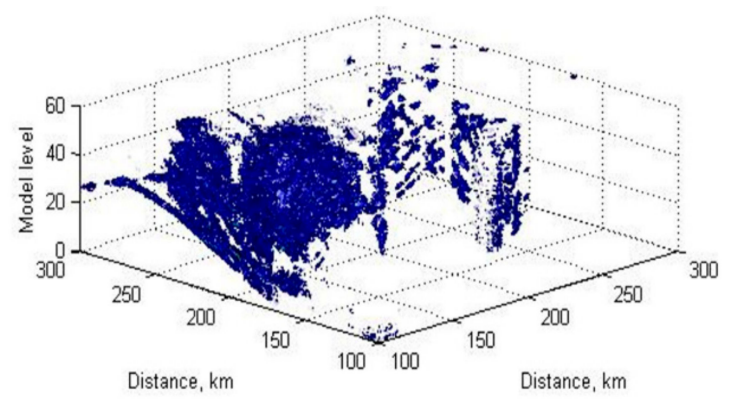

(b)

Figure 2. Cont. 


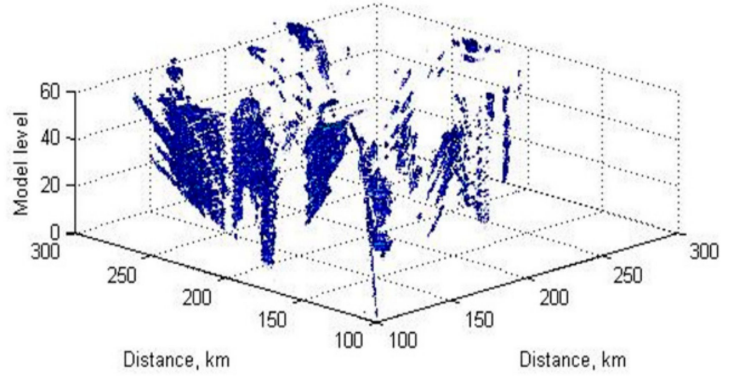

(c)

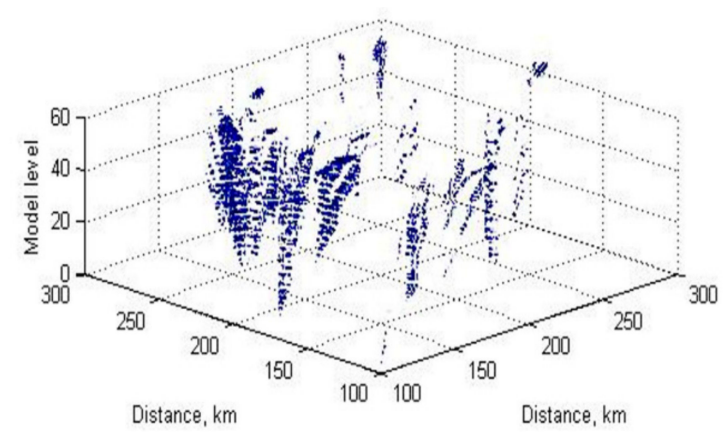

(d)

Figure 2. Filling-in the model grid by radar measurements depending on pre-processing smoothing parameters: (a) $0.5 \mathrm{~km},(\mathbf{b}) 1 \mathrm{~km},(\mathbf{c}), 2.5 \mathrm{~km}$, and (d) $5 \mathrm{~km}$.

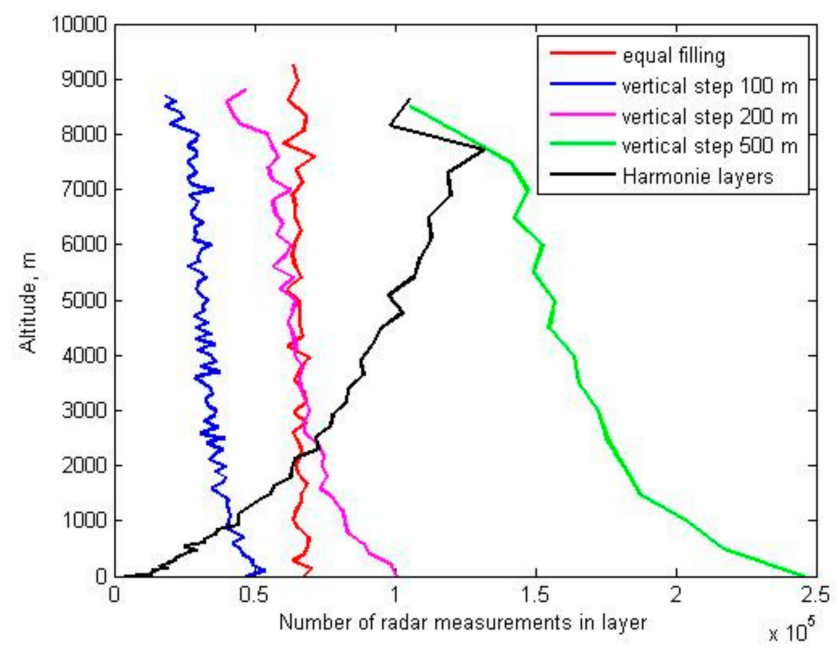

Figure 3. Filling-in the layers by radar data depending on the type of vertical step lists.

\subsection{Configuration of Radar Data Assimilation Experiments}

Radar reflectivity data from the BaltEx experiment covering the Finnish domain were used in this study (www.baltex-research.eu). The BaltRad project (se.baltrad.eu) related to these data points operates with high-quality data and has demonstrated to possess a significant value for forecasters and decision-makers. The Finnish domain was chosen due to several reasons. First, a major part of the domain is covered by radar measurements even though their inhomogeneous distribution is a factual issue. Second, the smooth orography and relatively homogeneous surface significantly decrease a potential external impact from sharp gradients of the complex orography and surface contrasts.

The experimental design has been implemented on the operational Harmonie platform as follows. A heavy precipitation event over Finland during 14-15 August 2010 with the maximum rain rate during the period 15:00-18:00 UTC was considered. Numerical experiments were performed in the framework of the mesoscale Harmonie-40h1.1 model with $2.5 \mathrm{~km}$ domain resolution. Three runs have been carried out with the same model configuration including 3D-Var data assimilation with a three-hour updating cycle. The differences between experiments were in only the radar data involvement and the pre-processing procedure. The control run (CNTR) included the assimilation of all the available SYNOP, TEMP, and AIREP but RADAR observation types over the domain. Two radar reflectivity data assimilation runs used the following approaches. The first approach proposes "cube-smoothing" (FINE) described above with the horizontal resolution equal to the model's $2.5 \mathrm{~km}$ resolution and vertical resolution, according to quasi-equivalent filling. The second one (COARSE) explores the operationally implemented procedure based on double subsequent smoothing of the radar network with resolutions of $8 \mathrm{~km}$ and $15 \mathrm{~km}$, respectively, while the model resolution was still kept to $2.5 \mathrm{~km}$ [14]. 


\section{Results of Radar Data Assimilation}

The results of the numerical experiments have revealed a noticeable impact of radar reflectivity data assimilated in the model on the prediction of precipitation patterns and rates. Moreover, it has been shown that this impact varies depending on pre-processing procedures and their corresponding parameters. Figure 4 shows the spatial distribution of precipitable water and its differences in experiments over the Finnish domain for 18:00 UTC 14 August 2010. This variable was chosen since it better pronounces continuous distribution of water in the atmosphere compared to the discrete form of surface precipitation (Figure 5). Even though both variables reflect major features of spatial coverage, certain discrepancies can be associated with the resolution in radar data assimilation (RDA) formulations. In particular, both methods of RDA lead to redistribution of precipitation toward fine-scale cells within large areas accompanied by an enlarging area of increased precipitation in total. The COARSE run provides larger amounts of precipitation than the FINE run. However, special attention would be paid to the South Finland area where six radars (Anjalankoski, Ikaalinen, Korpo, Kuopio, Vantaa, and Vimpeli (en.ilmatieteenlaitos.fi/fmi-radar-network)) account for the dense three-dimensional coverage of the water content field in the atmosphere. Analysis of the CNTR run with the operationally used configuration shows a rather homogeneous field of precipitable water over this area (see Figure 4a). RDA allows us to specify and redistribute precipitable water among several areas since it is displayed in Figure 4. The first area is associated with the increased precipitation rate in both RDA runs (area I in Figure 4b,c) while the gain is sufficiently higher in the FINE run. This is reflected by the negative values in Figure $4 \mathrm{~d}$ (COARSE-FINE runs). Over the second area (area II in Figure $4 b, c)$, both RDA runs decrease the water content in the atmosphere, which is actually reallocated toward the first neighboring one. As in the previous site, changes are larger in the FINE run, which result in the area of positive values in Figure $4 \mathrm{~d}$. A belt of lower precipitable water amount in the FINE run corresponds to the third area (area III in Figure $4 b, c)$, which, however, does not appear in the COARSE run.

Bearing in mind the above, it can be stated that RDA affects the prognostic fields of the precipitation rate and spatial distribution. In general, this leads to slightly increasing in total precipitation amount over the domain while redistribution of water in the atmosphere occurs in a form of fine-scale cells of opposite signs within a common area of precipitation. As expected, the value and size of these changes depend on the smoothing parameter. The larger this parameter, the smoother the impact in spatial coverage is and the smaller the deviations from the analysis are [21]. Matching the radar resolution to that of the model should minimize numerical effects while focusing on physical features. In particular, the belt and cell features in precipitation fields $[22,23]$ are better simulated with RDA. Higher vertical resolution used in "cube-smoothing" RDA allows us to correct the position of the condensation level, which reduces the phase error. However, the impact from RDA is numerically sensitive to the radar pre-processing approaches and their internal parameters. 


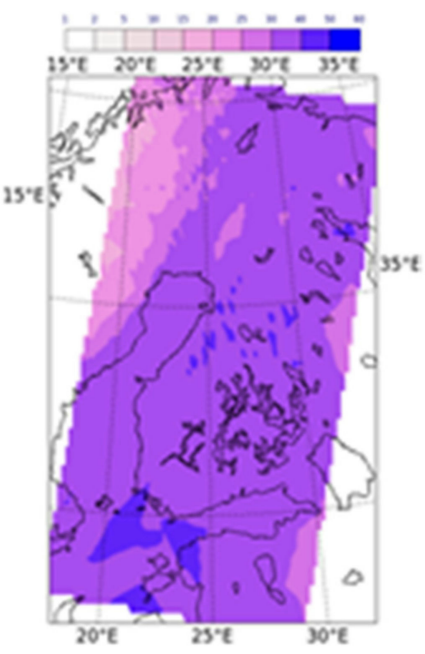

(a)

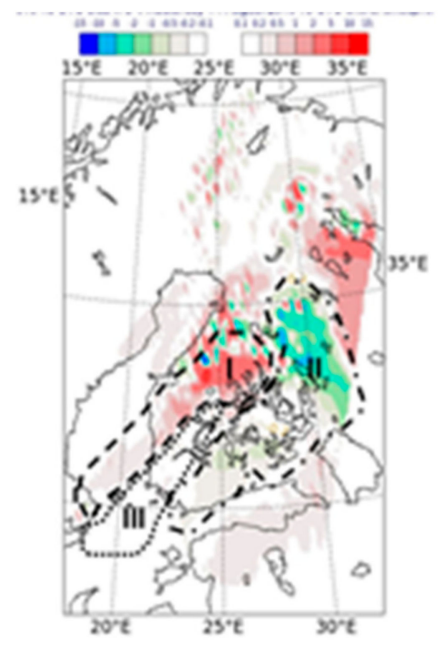

(c)

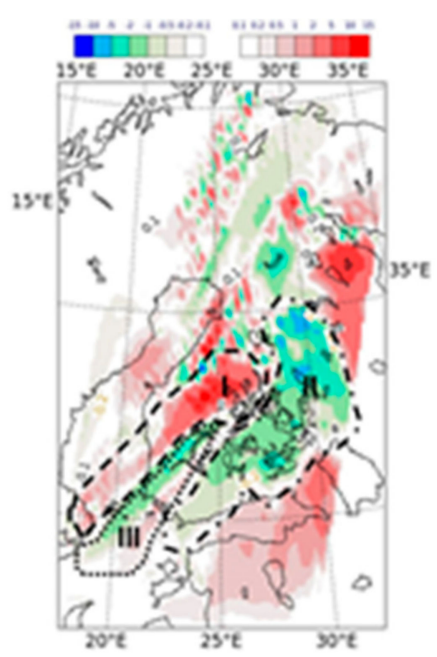

(b)

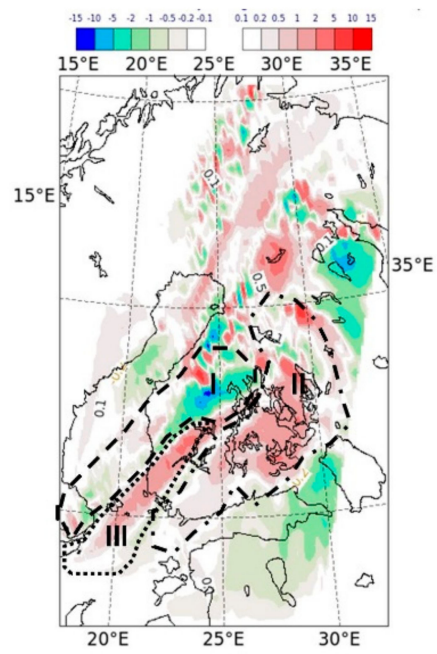

(d)

Figure 4. Spatial distribution of precipitable water over the Finnish domain for 18:00 UTC 14 August 2010. (a) in analysis and differences between runs: (b) FINE-CNTR, (c) COARSE-CNTR, (d) COARSE-FINE. Areas I, II, and III outline specific regions in redistributing of precipitable water.

The RDA procedures change also the vertical distribution of rain water in the atmosphere. Figure 6 shows the vertical profiles for this variable in the area of the largest differences between the FINE and COARSE runs. The former is similar, in its general features, to the control run, but slightly redistributes water from a 2.5 to a $3.5 \mathrm{~km}$ layer towards lower levels. This can produce rare ripe condensation and a decrease of downstream precipitation in favor of upstream, which is shown in Figure $4 \mathrm{~b}$. The COARSE run shows a significant reduction of water content in the area, which is explained by the following two reasons. The first is that the deep smoothing spreads a compact substance (water cell) over larger neighboring areas and decreases its concentration. The second, which is related to the above, is that the smoothing changes the water (vapor) concentration, which follows changes in a chain of microphysical processes. The detailed study of links in this chain is a subject of ongoing research. 


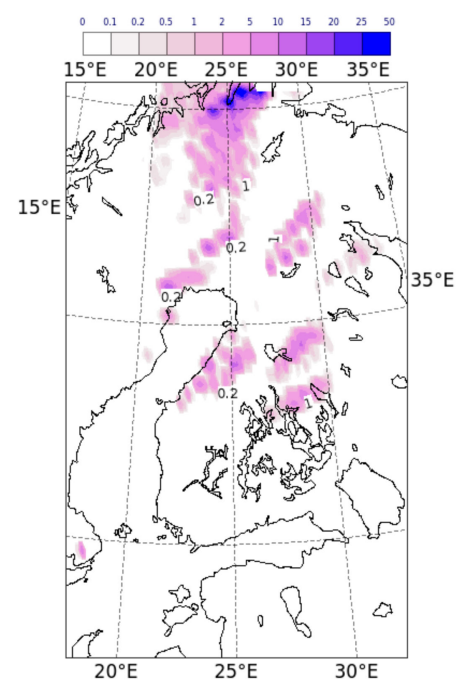

(a)

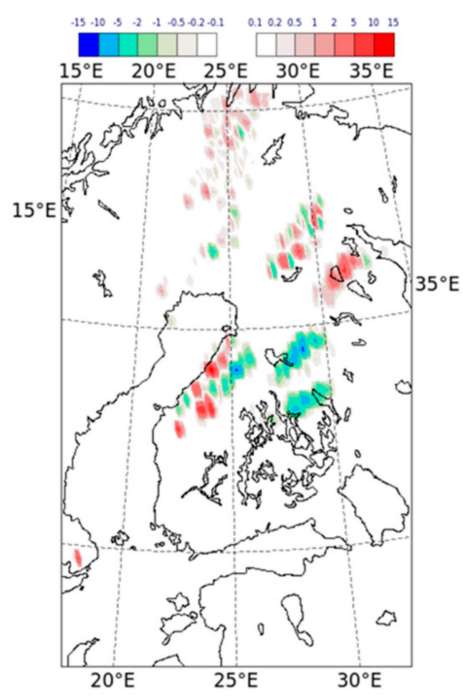

(c)

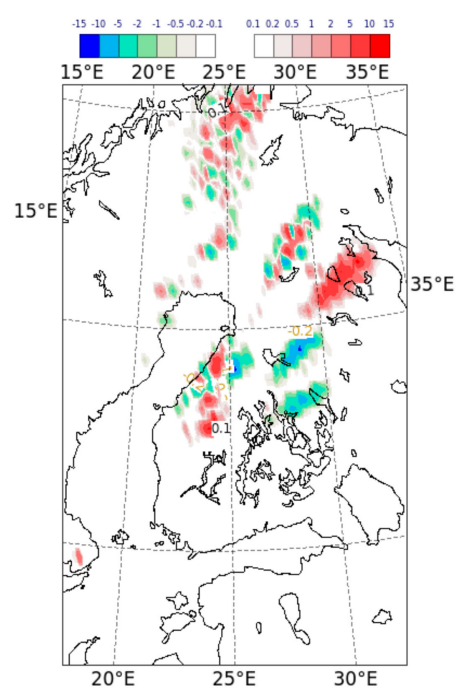

(b)

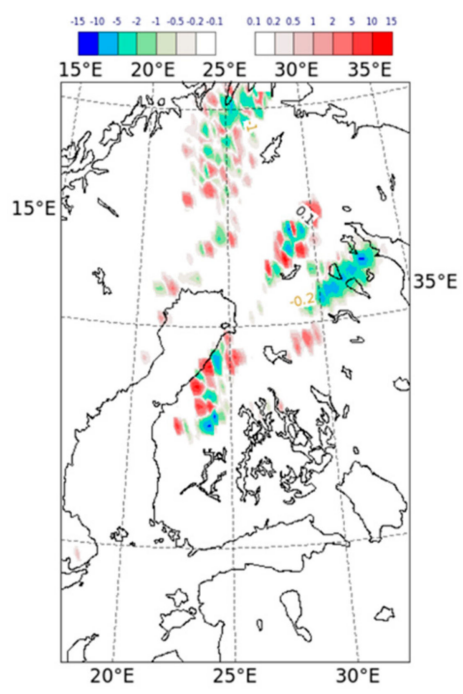

(d)

Figure 5. Same as Figure 4, but for surface precipitation.

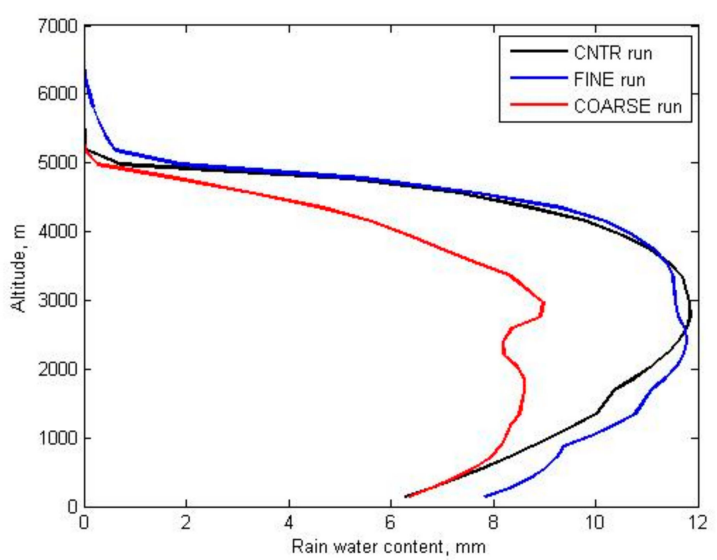

Figure 6. Vertical profiles of rain water in the atmosphere for different runs. 


\section{Discussion and Concluding Remarks}

The quality of numerical weather forecasts is crucially dependent on several factors. The data assimilation remains in the first line among these factors since this determines the error in the initial conditions and the further error growth rate during a forecast. The problem becomes even sharper for mesoscale atmospheric modeling with a resolution of order of a kilometer. Conventional data are less useful because of their coarse spatial and temporal resolutions. Remote sensing is a way for improving this situation due to the fact that it provides high resolution measurements of the order of a few hundred meters over the area of interest with a few minutes of a discretization time-step. However, another sort of problem appears in the forefront: this approach yields an abundance of information, which has to be screened from a useful core. Thus, a compromise has to be made between a volume of detailed information and a restricted amount of selected data, which, however, should still keep spatial and temporal atmospheric features. This is a subject that is dealt with the preprocessing step before data are forwarded to the assimilation module of a numerical atmospheric model.

In this research, the data assimilation system in the convection-permitting Harmonie model has been further developed by involving radar reflectivity measurements. The focus has been on optimizing the inner parameters of the pre-processing procedures. In pursuing the compatibility between the model resolution and smoothed radar observation density, the "cube-smoothing" approach has been proposed, which promotes the presentation of radar measurements on a regular grid at a resolution equal to that of the model's grid. This ensures the equivalent presentation of precipitation (reflectivity) structures in both spaces such as the model and observation in the sense of equally preserving the scales of precipitation patterns. From the spectral point of view for spatial frequencies (wave numbers), this implies an equal cut-off of a high-frequency band in the model and in the observation fields. In this way, at least one component of the forecast error associated with the representativeness error [17] has been minimized.

As shown from the results presented above, the numerical experiments with the proposed approach exhibit better simulation of mesoscale cells and belts of precipitation versus the respective control runs. In particular, instead of one large area with smoothed values for rain rates, the improved assimilation approach was allowed to specify and redistribute precipitable water among several sub-areas. Two of them have higher accumulated water content. The third area located between these has lost some precipitable water. Such redistribution retrieves mesoscale features with sizes from a few to ten kilometers in precipitation fields and improves rain rates over the domain. However, precise verification still remains an issue due to several reasons including being the instrumental error and transient functions for radar measurements as well as the dominating sizes of precipitation patterns in particular regions and under certain atmospheric flow regimes. The latter will be the focus of further studies.

Author Contributions: S.I. contributed to the conceptualization, methodology, formal analysis, investigation, and writing; S.M. contributed to the supervision, methodology, writing, review and editing; I.R. contributed to the formal analysis, investigation and visualization.

Funding: This research received no external funding.

Acknowledgments: The research was realized under support of the Enviro-PEEX on ECMWF project. The authors thank Alexander Mahura (FMI), who is the project principal investigator, for facilitating our involvement in the project and Mats Dahlbom (DMI), who is the author of the preporepapy script, for fruitful discussions.

Conflicts of Interest: The authors declare no conflicts of interest. 


\section{References}

1. Gao, J.; Tang, G. Similarities and improvements of GPM Dual-Frequency Precipitation Radar (DPR) upon TRMM precipitation radar (PR) in global precipitation rate estimation, type classification and vertical profiling. Remote Sens. 2017, 9, 1142. [CrossRef]

2. Ma, Y.; Zhang, Y.; Yang, D.; Farhan, S.B. Precipitation bias variability versus various gauges under different climatic conditions over the Third Pole Environment (TPE) region. Int. J. Climatol. 2015, 35, 1201-1211. [CrossRef]

3. Bellerby, T.; Todd, M.; Kniveton, D.; Kidd, C. Rainfall estimation from a combination of TRMM precipitation radar and GOES multispectral satellite imagery through the use of an artificial neural network. J. Appl. Meteorol. 2000, 39, 2115-2128. [CrossRef]

4. Bai, L.; Shi, C.; Li, L.; Yang, Y.; Wu, J. Accuracy of CHIRPS satellite-rainfall products over mainland China. Remote Sens. 2018, 10, 362. [CrossRef]

5. Kidd, C.; Bauer, P.; Turk, J.; Huffman, G.; Joyce, R.; Hsu, K.-L.; Braithwaite, D. Intercomparison of high-resolution precipitation products over Northwest Europe. J. Hydrometeorol. 2012, 13, 67-83. [CrossRef]

6. Huuskonen, A.; Saltikoff, E.; Holleman, I. The operational weather radar network in Europe. Bull. Am. Meteorol. Soc. 2014, 95, 897-907. [CrossRef]

7. Ducrocq, V.; Lafore, J.-P.; Redelsperger, J.-L.; Orain, F. Initialization of a fine-scale model for convective-system prediction: A case study. Q. J. R. Meteorol. Soc. 2000, 126, 3041-3065. [CrossRef]

8. Ducrocq, V.; Ricard, D.; Lafore, J.-P.; Orain, F. Storm-scale numerical rainfall prediction for five precipitating events over France: On the importance of the initial humidity field. Weather Forecast. 2002, 17, 1236-1256. [CrossRef]

9. Richard, É.; Cosma, S.; Tabary, P.; Pinty, J.-P.; Hagen, M. High-resolution numerical simulations of the convective system observed in the Lago Maggiore area on the 17 September 1999 (Map IOP 2a). Q. J. R. Meteorol. Soc. 2003, 129, 543-564. [CrossRef]

10. Lorenc, A.C. Analysis methods for numerical weather prediction. Q. J. R. Meteorol. Soc. 1986, 112, 1177-1194. [CrossRef]

11. Seko, H.; Kawabata, T.; Tsuyuki, T.; Nakamura, H.; Koizumi, K. Impacts of GPS-derived water vapor and radial wind measured by Doppler radar on numerical prediction of precipitation. J. Meteorol. Soc. Jpn. 2004, 82, 473-489. [CrossRef]

12. Salonen, K.; Järvinen, H.; Haase, G.; Niemelä, S.; Eresmaa, R. Doppler radar radial winds in HIRLAM. Part II: Optimizing the super-observation processing. Tellus 2009, 61A, 288-295. [CrossRef]

13. Desroziers, G.; Ivanov, S. Diagnosis and adaptive tuning of information error parameters in a variational assimilation. Q. J. R. Meteorol. Soc. 2001, 127, 1433-1452. [CrossRef]

14. Caumont, O.; Ducrocq, V.; Watterlot, É.; Jaubert, G.; Pradier-Vabre, S. 1D+3DVar assimilation of radar reflectivity data: A proof of concept. Tellus 2010, 62A, 173-187. [CrossRef]

15. Jung, Y.; Xue, M.; Tong, M.J. Ensemble Kalman filter analyses of the 29-30 May 2004 Oklahoma tornadic thunderstorm using one-and two-moment bulk microphysics schemes, with verification against polarimetric radar data. Mon. Weather Rev. 2012, 140, 1457-1475. [CrossRef]

16. Gao, J.; Xue, M.; Stensrud, D.J. The development of a Hybrid EnKF-3DVAR algorithm for storm-scale data assimilation. Adv. Meteorol. 2013, 2013, 12. [CrossRef]

17. Ivanov, S.; Palamarchuk, J. Fine-scale precipitation structure of a cold front and the problem of the representativeness error. Adv. Geosci. 2007, 10,3-8. [CrossRef]

18. Thepaut, J.-N.; Courtier, P.; Belaud, G.; Lemaitre, G. Dynamical structure functions in a four-dimensional variational assimilation: A case study. Q. J. R. Meteorol. Soc. 1996, 122, 535-561. [CrossRef]

19. Rabier, F. Overview of global data assimilation developments in numerical weather-prediction centres. $Q$. J. R. Meteorol. Soc. 2005, 131, 3215-3233. [CrossRef]

20. Ivanov, S.; Simmer, C.; Palamarchuk, J.; Bachner, S. The systematic error of precipitation and humidity in the MM5 model. Adv. Geosci. 2008, 16, 97-107. [CrossRef]

21. Nogueira, S.M.C.; Moreira, M.A.; Volpato, M.M.L. Evaluating precipitation estimates from Eta, TRMM and CHRIPS Data in the south-southeast region of Minas Gerais State-Brazil. Remote Sens. 2018, 10, 313. [CrossRef] 
22. Tweedy, D.L. Mesoscale precipitation patterns in extratropical cyclones and implications for cyclone development. J. Geophys. Res. Atmos. 1990, 95, 1987-1997. [CrossRef]

23. Wong, M.; Skamarock, W.C. Spectral characteristics of convective-scale precipitation observations and forecasts. Mon. Weather Rev. 2016, 144, 4183-4196. [CrossRef] 